inflammation and autoimmunity and aberrant fibroblast activation with excessive matrix deposition. We increasingly recognize that physiological wound healing and the initial stages of SSc share many commonalities. However, in contrast to physiological repair, these tissue repair programs are not effectively terminated in SSc resulting in progressive matrix deposition and tissue damage. The mechanisms of fibroblast activation may change during the cause of SSc; Profibrotic mediators released from infiltrating leukocytes, activated endothelial cells and degranulated platelets may drive fibroblast activation and collagen release in particular in early stages, whereas endogenous activation loops with activation of fibroblasts by epigenetic changes and biomechanical or physical factors may be of increasing importance in later stages of SSc.

In my presentation, I will discuss novel insights into the pathogenesis of SSc with a particular focus on the interplay of inflammation and fibroblast activation. I will focus on translational aspects and highlight mechanisms with high potential for transfer from bench-to-bedside.

Disclosure of Interests: None declared

DOI: 10.1136/annrheumdis-2019-eular.8460

\section{SP0112 IMMUNOSUPPRESSION - ONE FITS ALL VS. INDIVIDUALIZED SELECTION?}

Marco Matucci-Cerinic. University of Florence, Division of Rheumatology AOUC, florence 50139, Italy

Systemic sclerosis (SSc) is an autoimmune disease characterised by skin, vascular and internal organ involvement leading eventually to tissue fibrosis and atrophy. The best control of the disease is usually achieved in the early phase which is characterised by a diffuse tissue inflammation and vasculopathy. of the tissues. In this phase an immunosppressive stragey can in fact lead to disease remission even if some cases may escape control and progress inesorably to fibrosis.

Classically, the clinical profile of the patient needs to be achieved. The subset, the disease activity and the main clinical features should be defined in order to have the precise profile of the disease. The knowledge of the clinical conditions may therefore help in deciding the drug and shaping the therapeutic regimen and its intensity. Usually, the use of Cyclophosphamide (CYC) and Micophenolate (MMF) may fit the necessity of the largest part of the SSc population. In practice, CYC may be employed as an induction treatment, eithe rorally or intravenous, while MMF may be used as a maintenance therapy. Both drugs have been demonstrated to be useful in the treatment of SSc either on the cutaneous or on the lung involvement.

However, the most severe and refractory cases may be today considered for a hematopietic stem cell transplantation (HSCT). In this case, an individualised selection is performed before a patient can be considered fit to receive this kind of therapeutic regimen. Therefore, the treatment is strictly individualised and only after having a thorough clinical evaluation the patient can be accepted for the systemic conditioning with high doses of CYC for bone marrow ablation. In conclusion, the treatment of SSc is today mainly centered on immunesuppression whose intensity must be decided and individualized according to the disease activity. New innovative targeted drugs are on the horizon in the effort to find the right immunosuppressive and antifibrotic therapeutic approach clinically tailored on the SSc patient.

Disclosure of Interests: Marco Matucci-Cerinic Grant/research support from: Actelion, MSD, Pfizer, BMS, Chemomab, Sanipedia, Speakers bureau: Actelion, BMS; MSD, Janssen

DOI: 10.1136/annrheumdis-2019-eular.8534

\section{SP0113 STEM CELL TRANSPLANTATION - ALL SCIENTIFIC QUESTIONS ANSWERED?}

Jacob M. van Laar. University Medical Center Utrecht, Netherlands

Hematopoietic stem cell transplantation (HSCT) currently is the only disease modifying treatment for patients with poor prognosis diffuse cutaneous systemic sclerosis (dcSSc) based on three controlled clinical trials (ASSIST, ASTIS, SCOT). Nevertheless uptake of this intensive treatment modality is slow in most countries, due to funding issues, lack of medical experience, and concerns over toxicities including the risk of transplant-related mortality (TRM). Over the years, safety of HSCT has improved and TRM has dropped due to more stringent patient selection and better supportive care. Nevertheless, dcSSc remains a high-risk condition for $\mathrm{HSCT}$, and even a minimum degree of risk remains unavoidable. The decision to opt for HSCT therefore requires a thorough discussion of potential benefits and possible risks with a patient and their family and ultimately hinges on shared decision making and informed consent. Apart from such clinical issues there are still plenty of scientific questions to be answered. First, should HSCT be offered as firstline immunosuppressive or chemotherapy in high-risk patients, or is it better to reserve HSCT as salvage-therapy for those who failed a course of routine immunosuppression or i.v. pulse cyclophosphamide? And if so, on the basis of what criteria should treatment intensity be stepped up? Second, how can patient selection be optimised so as to avoid transplanting dcSSc patients with little chance of responding? Third, is there a window of opportunity where the immune system can be rebooted? Last but not least, how can potentially eligible dcSSc patients get access to HSCT? The latter may be the most (de)pressing one, as HSCT is only available in a small number of centres. Until efficacious disease-modifying drugs with a better risk-benefit ratio become available, HSCT will continue to be the only therapeutic option to reverse the disease course in dcSSc patients.

Disclosure of Interests: Jacob M. van Laar Grant/research support from: Genentech, Consultant for: F. Hoffmann-La Roche DOI: 10.1136/annrheumdis-2019-eular.8611

FRIDAY, 14 JUNE 2019

13:30:00 - 15:00:00

Predicting short-term fracture risk: can we foresee the (close) future?

\section{SP0114 CASE 1 PRESENTER: IMMINENT FRACTURE RISK ASSESSMENT AND DOES IT HELP CLINICAL MANAGEMENT}

Nicholas Fuggle. University of Southampton, MRC Lifecourse Epidemiology Unit, Southampton, United Kingdom

A 60 year old female was under long-term follow-up for rheumatoid arthritis, for which she took methotrexate. Ten years previously she had been found to have a single vertebral fracture and her fracture risk, assessed using The WHO Fracture Risk Assessment Tool (FRAX ${ }^{\circledR}$ ), was calculated as a $13 \%$ risk of major osteoporotic fracture over the next 10 years. According to The National Osteoporosis Guideline Group (NOGG) recommendations, she underwent Dual X-ray Absorptiometry (DXA) which demonstrated a bone mineral density T-score of -1.7 at the femoral neck. Lifestyle, vitamin $D$ and calcium intake were optimised and followup was arranged for 6 months.

Prior to her next appointment she fell and sustained a fracture of the distal radius. On fracture liaison review her FRAX ${ }^{\circledR}$ risk of fracture was $14 \%$ (rising due to an increased year of age) despite the recent change in clinical circumstances.

This case serves to emphasise the clinical conundra associated with imminent fracture risk (the 2 years after a fracture when the patient is at a significantly higher risk of fracture) and the difficulties with the decision to treat.

Disclosure of Interests: None declared

DOI: 10.1136/annrheumdis-2019-eular.8481

\section{SP0115 CASE 1 DISCUSSANT: IMMINENT FRACTURE RISK: ASSESSMENT AND DOES IT HELP CLINICAL MANAGEMENT}

Cyrus Cooper. University of Southampton, MRC Lifecourse Epidemiology Unit, Southampton, United Kingdom

With an estimated 520,000 fragility fractures every year in the UK, delivering effective and efficient healthcare for this patient group has significant consequences for patients, families, the NHS, and society. A fragility fracture is a major risk factor for further fractures, and healthcare systems are now beginning to recognise the benefits of secondary fracture prevention. Despite this, less than $50 \%$ of patients receive effective secondary fracture prevention after a fragility fracture. This has led to national and international initiatives to improve clinical services by implementing fracture liaison services (FLSs). Successful funding of a new FLS is usually influenced by the number of fractures it is expected to prevent in the first few years after an index fracture. The expected number of fractures prevented is in turn determined by the baseline risk of subsequent fracture, the number of patients at high enough fracture risk to warrant anti-osteoporosis medication (AOM), and the degree of fracture risk reduction by AOMs. Underestimating fracture risk in the post-fracture period will lead to fewer expected fractures prevented and lower perceived benefit of the FLS by payers, and importantly also by patients, families, healthcare providers, and payers. Tools are available to determine the long-term risk of fracture based on patient factors, including previous fracture. Of these, FRAX and QFracture have been incorporated within UK NICE clinical guidance, and the FRAX-derived intervention threshold is used to guide recommendations for AOM in the NHS.

Disclosure of Interests: Cyrus Cooper Consultant for: Personal fees from Alliance for Better Bone Health, Amgen, Eli Lilly, GSK, Medtronic, Merck, Novartis, Pfizer, Roche, Servier, Takeda and UCB.

DOI: 10.1136/annrheumdis-2019-eular.8450 